\title{
Momentum Distribution for Bosons with Positive Scattering Length in a Trap
}

\author{
T.T. Chou ${ }^{1}$ Chen Ning Yang ${ }^{2}$ and L.H. Yu ${ }^{3}$ \\ ${ }^{1}$ Department of Physics, University of Georgia, Athens, Georgia 30602 \\ ${ }^{2}$ Institute for Theoretical Physics, State University of New York, \\ Stony Brook, New York 11794 \\ ${ }^{3}$ National Synchrotron Light Source, Brookhaven National Laboratory, Upton, \\ New York 11973
}

\begin{abstract}
The coordinate-momentum double distribution function $\rho(\mathbf{r}, \mathbf{p}) d^{3} r d^{3} p$ is calculated in the local density approximation for bosons with positive scattering length $a$ in a trap. The calculation is valid to the first order of $a$. To clarify the meaning of the result, it is compared for a special case with the double distribution function $\rho_{w} d^{3} r d^{3} p$ of Wigner.
\end{abstract}


Using the local density approximation (LDA) $[1,2]$, which is a straightforward adaptation of the Thomas-Fermi method, the density distribution $\rho(\mathbf{r}) d^{3} r$ in coordinate space for BEC for $a>0$ in a trap has been obtained. We want to calculate in this paper the coordinate-momentum distribution $\rho(\mathbf{r}, \mathbf{p}) d^{3} r d^{3} p$ in the same approximation. We follow the notation of Ref. [2] throughout. In particular, the fugacity $z$ of the system is

$$
z=\exp [\mu / k T]
$$

where $\mu$ is the chemical potential. We introduce a local fugacity $\zeta(\mathbf{r})$ defined as

$$
\zeta=z \exp [-\beta V(\mathbf{r})]
$$

\section{The Gaseous Phase}

By the gaseous phase we include both the system before BEC sets in, and the system at high densities for the cells outside of $r_{0}$ [2], i.e., outside of the region where BEC takes place. We consider such a cell of volume $V$ in which the local fugacity is $\zeta$. Using the method of Ref.[3] we write the grand partition function in the cell as

$$
\mathcal{Q}=\sum_{N} \zeta^{N} \operatorname{Tr}\left[\exp \left(-\beta H_{0}-\beta H^{\prime}\right)\right] .
$$

The average occupation number $\ll n_{k} \gg$ of the state with momentum $\hbar \mathbf{k}$ can be computed from

$$
\mathcal{Q} \ll n_{k} \gg=\sum_{N} \zeta^{N} \operatorname{Tr}\left[\left(a_{k}^{\dagger} a_{k}\right) \exp \left(-\beta H_{0}-\beta H^{\prime}\right)\right] .
$$

We shall drop all terms beyond the first order of $H^{\prime}$. Since $H_{0}$ commutes with $a_{k}^{\dagger} a_{k}$, we find

$$
\mathcal{Q}=\sum_{N} \zeta^{N} \operatorname{Tr}\left[\exp \left(-\beta H_{0}\right)\left(1-\beta H^{\prime}\right)\right]=\mathcal{Q}_{0}+\mathcal{Q}_{1}
$$

and

$$
\mathcal{Q} \ll n_{k} \gg=\sum_{N} \zeta^{N} \operatorname{Tr}\left[\exp \left(-\beta H_{0}\right)\left(a_{k}^{\dagger} a_{k}\right)\left(1-\beta H^{\prime}\right)\right]=\mathcal{A}_{0}+\mathcal{A}_{1}
$$

where

$$
\mathcal{Q}_{0}=\sum_{N} \zeta^{N} \operatorname{Tr}\left[\exp \left(-\beta H_{0}\right)\right]=\prod\left[1-\zeta e^{-\beta \varepsilon}\right]^{-1}
$$


is the term in $\mathcal{Q}$ without the perturbation term. $\mathcal{Q}_{1}$ has been evaluated in Ref.[3].

$$
\mathcal{Q}_{1} / \mathcal{Q}_{0}=-\beta \frac{4 \pi a \hbar^{2}}{m V}\left[\sum_{\alpha \neq \beta} \bar{n}_{\alpha} \bar{n}_{\beta}+\sum_{\alpha} \frac{1}{2} \overline{n_{\alpha}^{2}}-\sum_{\alpha} \frac{1}{2} \bar{n}_{\alpha}\right]
$$

where the bar means average over the grand canonical ensemble $\mathcal{Q}_{0}$ :

$$
\bar{n}_{\alpha}=\frac{\zeta e^{-\beta \varepsilon_{\alpha}}}{1-\zeta e^{-\beta \varepsilon_{\alpha}}}
$$

Similarly

$$
\mathcal{A}_{0} / \mathcal{Q}_{0}=\bar{n}_{k}
$$

and

$$
\mathcal{A}_{1} / \mathcal{Q}_{0}=-\beta \frac{4 \pi a \hbar^{2}}{m V}\left\langle n_{k}\left[\sum_{\alpha \neq \beta} n_{\alpha} n_{\beta}+\sum_{\alpha} \frac{1}{2} n_{\alpha}^{2}-\sum_{\alpha} \frac{1}{2} n_{\alpha}\right]\right\rangle
$$

where the symbol $\langle>$ means the same average as the bar. The coefficient in (8) and (11), $-\beta 4 \pi a \hbar^{2}(m V)^{-1}$, is equal to $-2 a \lambda^{2} V^{-1}$. Now (11) can be rewritten as

$$
\mathcal{A}_{1} / \mathcal{Q}_{0}=-2 a \lambda^{2} V^{-1}\left\langle\left(n_{k}-\bar{n}_{k}\right)\left[\sum_{\alpha \neq \beta} n_{\alpha} n_{\beta}+\sum_{\alpha} \frac{1}{2} n_{\alpha}^{2}-\sum_{\alpha} \frac{1}{2} n_{\alpha}\right]\right\rangle+\bar{n}_{k} \mathcal{Q}_{1} / \mathcal{Q}_{0} .
$$

Notice that $\mathcal{Q}_{0}$ is a product distribution function according to (7). Thus $\bar{n}_{\alpha} n_{\beta}=\bar{n}_{\alpha} \bar{n}_{\beta}$ if $\alpha \neq \beta$. Using this and similar identities we find that in the sum over $\alpha$ and $\beta$ in (12), the bracket \langle\rangle vanishes unless $k=\alpha$ or $k=\beta$. Thus

$$
\mathcal{A}_{1} / \mathcal{Q}_{0}=-2 a \lambda^{2} V^{-1}\left\langle\sum_{\beta \neq k} 2 n_{k} n_{\beta}\left(n_{k}-\bar{n}_{k}\right)+\frac{1}{2}\left(n_{k}^{3}-\bar{n}_{k} n_{k}^{2}-n_{k}^{2}+\bar{n}_{k}^{2}\right)\right\rangle+\bar{n}_{k} \mathcal{Q}_{1} / \mathcal{Q}_{0} .
$$

Now $V^{-1}\left\langle\sum_{\beta \neq k} n_{k} n_{\beta}\left(n_{k}-\bar{n}_{k}\right)\right\rangle \rightarrow \rho\left(\overline{n_{k}^{2}}-\bar{n}_{k}^{2}\right)$ as $V \rightarrow \infty$, yielding

$$
\mathcal{A}_{1} / \mathcal{Q}_{0}=-4 a \lambda^{2} \rho\left(\overline{n_{k}^{2}}-\bar{n}_{k}^{2}\right)+\bar{n}_{k} \mathcal{Q}_{1} / \mathcal{Q}_{0}
$$

Adding this to $(10)$ and dividing by $1+\mathcal{Q}_{1} / \mathcal{Q}_{0}$ we obtain, to order $a$,

$$
\ll n_{k} \gg=\bar{n}_{k}-4 a \lambda^{2} \rho\left(\overline{n_{k}^{2}}-\bar{n}_{k}^{2}\right) .
$$

The number of modes $\mathbf{k}$ in a cell of volume $V$ is $\left(8 \pi^{3}\right)^{-1} V d^{3} k$. Thus the combined coordinate-momentum distribution $\rho(\mathbf{r}, \mathbf{p})$ is given by

$$
h^{3} \rho(\mathbf{r}, \mathbf{p})=\ll n_{k} \gg=\frac{\zeta e^{-\beta \varepsilon_{\alpha}}}{1-\zeta e^{-\beta \varepsilon_{\alpha}}}-4 \pi \lambda^{2} \rho(r) \frac{\zeta e^{-\beta \varepsilon_{\alpha}}}{\left(1-\zeta e^{-\beta \varepsilon_{\alpha}}\right)^{2}}
$$


where $\varepsilon_{k}=\frac{\hbar^{2} k^{2}}{2 m}$ and $\zeta$ is given by (2). In (16) we have evaluated $\overline{n_{k}^{2}}$ in a straightforward way from the product partition function (7).

Integrating (16) over $d^{3} p$ we should get the density $\rho(r)$ times $h^{3}$. This can be done without much difficulty, yielding Eq. (3) of Ref. [2].

\section{The Region with Condensate}

For high densities, BEC forms in some cells of the trap. In those cells $\rho=\rho_{0}+\rho_{s}>$ $\rho_{0}$, where [4],

$$
\rho_{0}=\lambda^{-3} g_{3 / 2}(1)
$$

and

$$
V(r)+4 \pi a \rho_{s}(r) \hbar^{2} / m=V\left(r_{0}\right) .
$$

Here $\rho_{s}$ denotes superfluid density, i.e., density of particles with $\mathbf{p}=0$. An important parameter $\xi_{5}=\rho_{s} / \rho$, a function of the location of the cell, with value between 0 and 1 , describes incomplete occupation of the ground state, and was studied in detail in Ref. [5]. [Notice that $\xi_{5}$ and $\xi$ are totally different quantities.] For cells without BEC, $\xi_{5}=0$.

It was shown in Ref. [5] that the system in a cell with BEC has an energy given by (5.16) with a phonon spectrum (for $k \neq 0$ ) given by (5.18):

$$
\hbar \omega_{k}=\frac{\hbar^{2}}{2 m}\left(k^{4}+2 k_{0}^{2} k^{2}\right)^{1 / 2}, \quad k_{0}^{2}=8 \pi a \xi_{5} \rho=8 \pi a \rho_{s} .
$$

Notice that for the gaseous phase, $\xi_{5}=0$ and the phonon spectrum is quadratic for small $k$.

The phonon creation operator $b_{k}^{\dagger}$ and the particle creation operator $a_{k}^{\dagger}$ are related to each other through a Bogoliubov transformation [6]:

$$
a_{k}=\left(b_{k}-\alpha_{k} b_{-k}^{\dagger}\right)\left(1-\alpha_{k}^{2}\right)^{-1 / 2}
$$

where

$$
\alpha_{k}=k_{0}^{-2}\left(k^{2}+k_{0}^{2}-\sqrt{k^{4}+2 k^{2} k_{0}^{2}}\right) .
$$


For a state with $m_{k}$ phonons we can compute the average occupation number $\ll n_{k} \gg$ of atoms in the state $\mathbf{p}=\hbar \mathbf{k}$ using (20) above. The result is linear in $m_{k}$. Now the average number of $m_{k}$ is given by Eqs.(5.27) and (5.31). Thus

$$
\rho(\mathbf{r}, \mathbf{p})=h^{-3}\left[\alpha_{k}^{2}+\left(1+\alpha_{k}^{2}\right)\left(e^{\beta \hbar \omega_{k}}-1\right)^{-1}\right]\left(1-\alpha_{k}^{2}\right)^{-1}, \quad(k \neq 0),
$$

where $\omega_{k}$ is given by (19), and $\alpha_{k}$ is given by (21).

For $k \gg k_{0}=\sqrt{8 \pi a \rho_{s}}$, the phonon energy (19) can be expanded in powers of $a$ and (22) becomes

$$
\rho(\mathbf{r}, \mathbf{p})=h^{-3}\left(e^{\beta E_{k}}-1\right)^{-1}, \quad\left(k \gg k_{0}\right),
$$

where

$$
E_{k}=\frac{p^{2}}{2 m}+\frac{\hbar^{2}}{2 m}\left[8 \pi a \rho_{s}(r)\right]
$$

For other values of $k>0$, Eq.(22) gives the distribution. It is a complicated function of $k$. For $0<k \ll k_{0}$, it reduces to

$$
\rho(\mathbf{r}, \mathbf{p}) \cong h^{-3} m \beta^{-1} p^{-2}, \quad\left(0<k \ll k_{0}\right)
$$

Notice that this differs by a factor of 2 from the corresponding distribution when $a=0$.

\section{Wigner Double Distribution}

What is the meaning of the double distribution $\rho(\mathbf{r}, \mathbf{p})$ ? It, of course, should only be used [2] for $d^{3} r>\left(L_{2}\right)^{3}$, and for $d^{3} r d^{3} p>h^{3}$. But does it have a clear meaning in quantum mechanics? We discuss this by examining Eq.(16) in the limit of $a=0$, for the case of a spherically symmetrical harmonic trap $V(r)=\frac{1}{2} m \omega^{2} r^{2}$. In such a case we can compute exactly the matrix element of $\left\langle\mathbf{r}^{\prime}\left|\frac{z e^{-\beta H}}{1-z e^{-\beta H}}\right| \mathbf{r}\right\rangle=\sum_{\ell=1}^{\infty}\left\langle\mathbf{r}^{\prime}\left|z^{\ell} e^{-\beta \ell H}\right| \mathbf{r}\right\rangle$, by using, e.g., the result of Ref.[9]. Using Wigner's idea [10], we put $\mathbf{r}^{\prime}=\mathbf{R}-\frac{1}{2} \eta$, and $\mathbf{r}=\mathbf{R}+\frac{1}{2} \eta$ and evaluate the above, and then make a Fourier transform to the variable $\mathbf{P}$ conjugate to $\eta$.

The resultant double distribution $a$ la Wigner becomes

$$
\rho_{w}(\mathbf{R}, \mathbf{P})=h^{-3} \sum_{\ell=1}^{\infty} z^{\ell}\left(\operatorname{sech} \frac{\ell \varepsilon}{2}\right)^{3} \exp \left\{-\frac{2 \beta}{\varepsilon}\left(\tan h \frac{\ell \varepsilon}{2}\right)\left(\frac{P^{2}}{2 m}+\frac{1}{2} m \omega^{2} R^{2}\right)\right\}
$$


where $\varepsilon=\beta \hbar \omega$. In the limit that $\varepsilon \rightarrow 0$, this is exactly Eq.(16) for $a=0$, [noticing that the local fugacity $\zeta$ is given by (2)] which is in agreement with the discussion in Ref. [2] for the single distribution function $\rho(r)$.

The work of CNY is supported in part by an NSF Grant PHY-9309888. That of LHY is performed under the auspices of US DOE. 


\section{REFERENCES}

1. J. Oliva, Phys. Rev. B39, 4197 (1989).

2. T. T. Chou, Chen Ning Yang and L. H. Yu, to appear in Phys. Rev. A.

3. K Huang, C. N. Yang and J. M. Luttinger, Phys. Rev. 105, 776 (1957).

4. In Ref. [2] we only discussed cases where $V(r)$ is spherically symmetrical. But it is obvious that the discussion can be extended to nonspherically symmetrical cases.

5. T. D. Lee and C. N. Yang, Phys. Rev. 112, 1419 (1958). To avoid confusion of notations between Refs. [2] and [5], we add a subscript 5 to the parameters $\xi$ and $\zeta$ of Ref. [5]. Furthermore we refer to equation x in Ref. [5] as (5.x).

6. N. N. Bogoliubov, J. Phys. USSR, XI, 23 (1947), first introduced the mathematical trick later known as the Bogoliubov transformation. But the concept of scattering length was not used and the physics of the paper was incorrect. In 1957 in Ref. [7] the physics of the dilute hard sphere boson system was reduced to a Hamiltonian problem solved in the appendix of that paper. Later it was pointed out [8] that this Hamiltonian problem can be more easily solved with the Bogoliubov transformation. We follow here this later method.

7. T. D. Lee, K. Huang and C. N. Yang, Phys. Rev. 106, 1135 (1959).

8. K. Huang, Statistical Mechanics (John Wiley, New York 1963).

9. R. P. Feynman, Statistical Mechanics: a Set of Lectures (Benjamin, New York, 1972).

10. E. Wigner, Phys. Rev. 40, 749 (1932). 